\section{Fending for themselves}

Ian Stewart

Fractais: An Interdisciplinary Journal on the Complex Geometry of Nature. Honorary editor B. B. Mandelbrot; managing editors M. Matsushita, M. Shlesinger and T. Vicsek. World Scientific. 4/yr. $\$ 240$ (institutional); $\$ 96$ (personal/institutions in developing countries).

OVER the past decade the subject of fractals has matured into a distinct area of scientific enquiry. Like all active research areas, its boundaries are fuzzy and its sub-

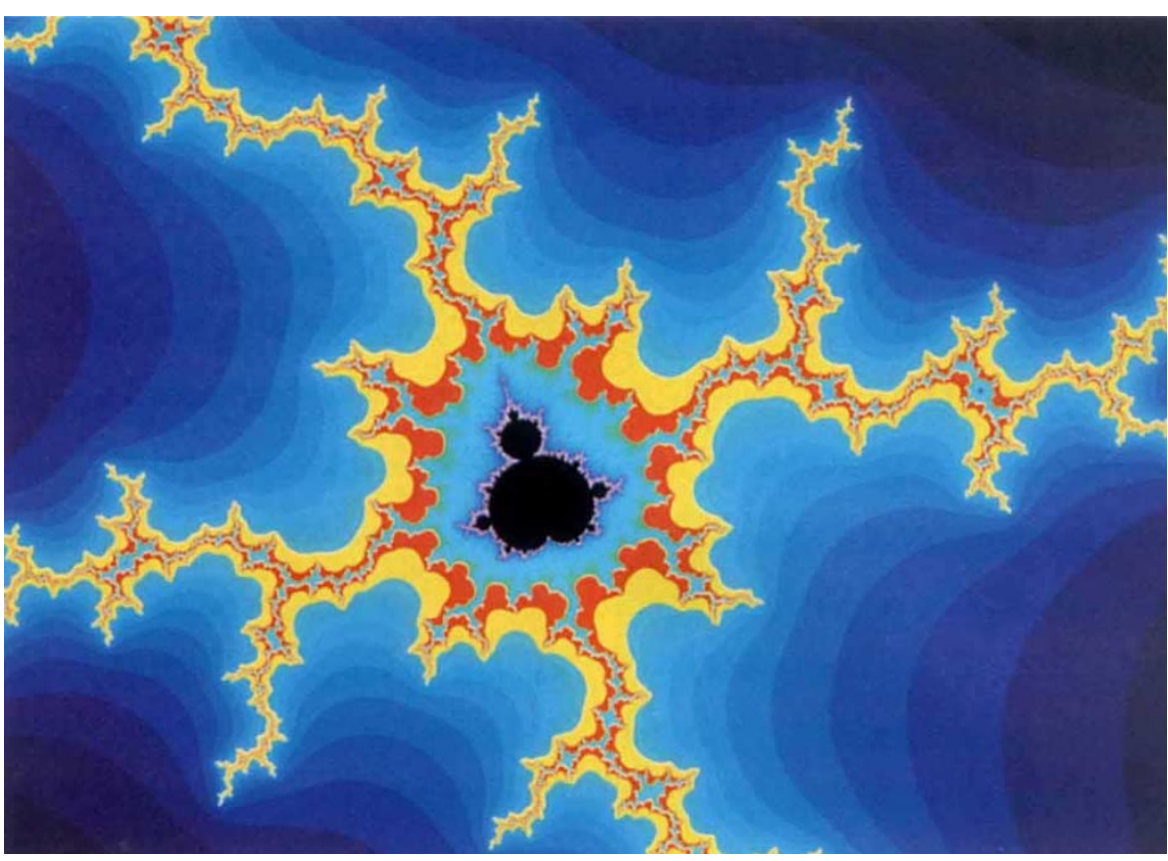

Fractal geometry - a computer graphics image entitled "A Birth of Lightning", derived from the Mandelbrot set.

ject matter is not always precisely defined. Mathematically, fractals are 'recursive geometry' - geometric forms with structure on all scales; physically they are natural phenomena with structure on an unusually wide range of scales. The mathematics is a model of reality, not a faithful representation - as usual.

As early as 1984, various publishers conceived the idea of a specialist journal devoted to fractals. Their creator, Benoît Mandelbrot, sensibly declined all invitations to edit such a journal on the grounds that it would be premature. The way to publish an interdisciplinary subject is to disperse it among all the journals of all the appropriate disciplines. Yet now we have a specialist journal. What has changed? Fractals have grown up, and it is time for the fledgling science to leave the nest and fend for itself.

Fractals is physically impressive: large format, glossy pages, profusely illustrated with occasional colour. Scientifically it is fairly impressive too, with an attractive mix of theory and experiment, simulations and proofs. The first two volumes already cover most of the main areas in which fractal geometry has become an accepted tool: fracture surfaces, aggregation processes, image compression, $1 / f$ noise. They also include papers in many new areas. A good example, by Eshel BenJacob and collaborators, discusses the complex patterns that form during the growth of colonies of bacteria. These structures range from tree-like branching forms to dendritic clusters to compact discs, and they are studied experimentally and by computer simulation. Somewhere towards the opposite end of the scientific spectrum are papers such as that by Lajos

$$
\text { have }
$$

In the past, the main motivation of many Russian authors seems merely to have been to stake their claim to a theorem, rather than to explain the ideas and methods underlying its proof. Articles were often excessively brief, with key steps and arguments sometimes entirely omitted. Also, the writing, translation and typesetting were generally rather poor, with frequent mistakes and typographical errors.

So the Russian Journal of Mathematical Physics is a welcome step in developing a uniform quality in the literature. The papers are of a high standard; in particular, they are generally extremely well written, carefully translated and clearly typeset (in the usual Western style). There has obviously been considerable effort to make them readable and easily understandable to nonspecialist mathematical and theoretical physicists. The intended audience is essentially the same as that catered for by the Joumal of Mathematical Physics, which the new journal should complement successfully.

"Due to the cardinal changes in world Nyikos and collaborators, who obtain numerical calculations of various fractal dimensions (box dimension, information dimension, mass exponent) of drawings by artists such as Picasso, Dürer, Rembrandt and Munch.

Several volumes are special issues of conference proceedings. For example, volume 2 number 3 is the proceedings of the "Fractals In Engineering" conference, held in 1994, which concentrates mainly on the use of fractal methods in image processing.

Fractals demonstrates the breadth, depth and vitality of the area, and it is essential reading for anybody whose research involves structures and forms that are irregular yet have a definite character of their own.

Ian Stewart is in the Department of Mathe matics, University of Warwick, Coventry CV4 7AL, UK. politics and the conversion of the military industry we will be able to publish papers that previously would have been contained in secret reports", claim the editors in the journal's stated aims and scope. I, for one, will certainly be curious to see the sort of results generated in this area by the remarkable levels of funding lavished on defence-related research over the past few decades.

Jon Keating is in the Department of Mathe matics, University of Manchester, Oxford Road, Manchester M13 9PL, UK.

\section{Autumn Books}

Nature's next review supplement is Autumn Books, which will appear in the issue of 17 November 1994. 